\title{
Von der Landeskunde zum interkulturellen Lernen - mit Werbung? ${ }^{1}$
}

\section{Thomas Stahl}

The article offers reflections on the use of advertising material in foreign language teaching. The focus is the potential of advertisement for cultural and intercultural learning due to the cultural determination.

Foreign language teaching - advertisement - cultural learning - intercultural learning

Der Beitrag bietet Überlegungen zum Einsatz von Werbematerial im Fremdsprachenunterricht. Im Fokus steht dabei das Potenzial von Werbung für landeskundliches und interkulturelles Lernen aufgrund ihrer kulturellen Determiniertheit.

Fremdsprachenunterricht - Werbung - Landeskundliches Lernen - Interkulturelles Lernen

Ohne Zweifel ist Werbung in unserer modernen Gesellschaft nicht nur zu einem bedeutenden Faktor des wirtschaftlichen Systems geworden. Werbung zeigt sich darüber hinaus als alltägliches und unsere Lebenswelt prägendes Phänomen und ist sicherlich ein einflussreicher Faktor der Sozialisation. Zwangsläufig präge sie sich, so stellt HURRELMANN $(2000,16)$ fest, unserem Wissen von den Dingen, unserem sprachlichen Repertoire und unserer Wirklichkeitswahrnehmung ein. Und für NORD $(1996,61)$ ist klar, dass Werbung ein wesentlicher „Bestandteil unserer Kultur" ist - und das in nahezu jeder Kultur. Allein diese Befunde bieten meiner Ansicht nach bereits eine ausreichende Rechtfertigung für den Einsatz von Werbung im Fremdsprachenunterricht, vor allem dann, wenn der Unterricht einer interkulturell ausgerichteten Fremdsprachendidaktik verpflichtet ist. Wenn nämlich Werbung tatsächlich „Bestandteil unserer Kultur“ ist, dann wird in ihr und durch sie auch Kultur präsentiert. Dazu kommt, dass für die Lerner Werbung - insbesondere im Hinblick auf Werbetechniken - ein bekanntes Kommunikationsmuster darstellt. In diesem Zusammenhang lässt sich KOLLMANN $(1998,18)$ zustimmen, wenn er schlussfolgert: „Werbung ist nicht nur ein fixer, immerfort gegenwärtiger Bestandteil der Lebenswelt der Menschen, sie ist auch ,global' geworden - Werbung ist in Zentralafrika ebenso anzutreffen, wie in Indonesien, Chile oder Österreich.“

Werbematerialien als Lernmedien lassen sich nach meiner Überzeugung in unterschiedlichen Kontexten des Fremdsprachenlernprozesses einsetzen und zahlreiche Lernarrangements sind im Umgang mit Werbung denkbar. Dies bestätigt

1 Dieser Aufsatz ist eine Zusammenfassung eines Vortrags, der im Juni 2009 an der Westböhmischen Universität Pilsen gehalten wurde. Aus technischen sowie insbesondere rechtlichen Schwierigkeiten wird auf die Wiedergabe der im Vortrag präsentierten Werbebeispiele verzichtet. Für weitere Informationen steht der Verfasser aber jederzeit zur Verfügung. 
auch ein Blick in die einschlägige Literatur und in aktuelle Unterrichtsmaterialien, wo sich Vorschläge für phonetische Übungen mit Hilfe von Werbeslogans ebenso wie zur Arbeit an Wortbildungsstrukturen oder grammatischen Kategorien anhand von Werbetexten finden. Auch Hörverstehensübungen anhand von Radio- oder Fernsehwerbespots ebenso wie Übungen zum Leseverstehen sind grundsätzlich vorstellbar. So halten LOHFERT/SCHWECKENDIEK (1989, Vorwort) gerade Werbeanzeigen für „besonders geeignet, um Strategien zum Entschlüsseln von fremdsprachlichen Texten einzuüben und die Lerner vom Wort-für-Wort-Lesenund-Verstehen-Wollen wegzubringen und sie zu einem flüssigen Erfassen von Texten [...] hinzuführen." Begründet wird diese Eignung mit dem Vorwissen der Lerner über die Textsorte und ihre Mitteilungsabsicht, das das Verstehen erheblich erleichtere. Werbung sei nämlich international und besitze eine uniforme Struktur. Auch würden mit Bildern und der Typografie bedeutsame Verständnishilfen in Werbeanzeigen angeboten.

Auffallend ist, dass sich die unterrichtspraktischen Anregungen nahezu ausschließlich auf die sprachbezogenen Anteile von Werbung beziehen. Kritisch anzumerken bleibt hier, dass eine rein sprachbezogene Arbeit mit Werbematerial im Fremdsprachenunterricht und eine didaktischen Bewertung, die den Nutzen von Werbung hauptsächlich in der Arbeit an und mit deren Sprache erkennt, nicht unproblematisch sind. Denn Schwierigkeiten können sich insbesondere aus der Sprache der Werbung selbst ergeben: Typische Merkmale der Werbesprache wie Sprach- und Wortspiele sowie Wortneubildungen, Abweichungen von grammatischen und orthografischen Normen, die Verwendung von Umgangssprache, der gehäufte und oft sprachspielerische Einsatz von Phraseologismen, aber auch intertextuelle Zitate, die häufig stark soziokulturell gebunden erscheinen, können das Verstehen deutlich erschweren und die Lerner verunsichern. Gerade der Bereich der Sprachinhalte, die in besonderem Maße kulturell-gesellschaftliche Prägung und Bindung aufweisen, kann zu Verstehensschwierigkeiten führen.

Darüber hinaus ist zu bedenken, dass die Sprache ihren Status als „Hauptmedium der Werbung“, den RÖMER $(1973,24)$ in den Anfangsjahren der Werbesprachforschung in den 1970er Jahren noch erkennen konnte, in der Gegenwart sicherlich verloren hat. Sprachelemente sind zwar nicht gänzlich irrelevant, aber in ihrer Bedeutung zu Gunsten visueller Anteile stark zurückgedrängt worden. Vor allem in Werbespots gehen die eigentlichen suggestiven Impulse vom Nichtsprachlich-Optischen aus, bisweilen auch von der Musik. Die sprachlichen Anteile beschränken sich dagegen oft auf den Markennamen und einen knappen, plakativen Slogan. MAIWALD $(2005,32)$ spricht in diesem Zusammenhang vom „Rückbau verbal gestützter Rhetorik zugunsten visuell getragener Ästhetik“. Aus diesem Grund müssen Unterrichtskonzepte, die rein auf die verbalen Elemente der Werbung bezogen bleiben, ins Leere laufen, will man im Unterricht zeitgemäße und aktuelle Werbung nutzen. 
Dieser kritische Einwand soll jedoch nicht auf die Empfehlung hinauslaufen, auf den Einsatz von Werbematerial generell zu verzichten. Lediglich eine Beschränkung auf die sprachlichen Anteile von Werbung ist didaktisch nicht (mehr) zu vertreten. Vielmehr müssen Unterrichtskonzepte über die Betrachtung der Sprache hinausgehen und die veränderten Textstrukturen der Werbung mit ihrem Ausbau von Visualität und dem Rückbau von Sprachlichkeit berücksichtigen. So gesehen bietet sich Werbung meines Erachtens hervorragend als Sprech- und Schreibanlass in vielerlei Hinsicht an. Werbung will Aufmerksamkeit erregen, will zu Lektüre und Betrachtung und natürlich zum Kauf anregen. Die der Werbung inhärente Aufforderung zum Kauf kann im Unterricht zur Aufforderung zum Sprechen oder Schreiben abgewandelt werden. Auch das hohe kreativ-produktive Potenzial von Werbung ist in diesem Zusammenhang ein entscheidender Faktor. Daraus ergeben sich vielfältige Anknüpfungspunkte für kreatives Sprechen oder Schreiben, die sich nicht nur mit fortgeschrittenen Lernern, sondern auch mit Lernern im Bereich der Grundstufe gewinnbringend nutzen lassen.

In einigen methodischen-didaktischen Anregungen zum Einsatz von Werbematerial im Fremdsprachenunterricht wird auch auf das Potenzial von Werbung im Hinblick auf das landeskundliche Lernen herausgestellt. So formulieren beispielsweise MACAIRE/HOSCH (1996, 138): „Da Werbung [...] immer landeskundlicheInformationen enthält [...], eignetsich Werbungbesondersgutfürden landeskundlichen Aspekt. "Dabei ist allerdings zu bedenken, dass Werbung sicherlich äußerst selten konkrete landeskundliche Fakten bietet oder eine lebensweltliche Realität abbildet, sondern in ihr eher Idealbilder und Wunschvorstellungen einer Gesellschaft sichtbar werden, die Rückschlüsse auf das vorherrschende Zeit- und Lebensgefühl erlauben. Landeskundliche Informationen enthält Werbung, indem sie als eine Art Seismograf Bewegungen im sozialen und politischen Klima in konzentrierter Form aufzeichnet (vgl. OCHMANN: 2001, 4) und zeigt, welche kollektiven Wertvorstellungen, Wunsch- und Leitbilder in der Gesellschaft, der sie entstammt, existieren. Mit Hilfe der Frage, woher diese Wünsche rühren, welche gesellschaftlichen, politischen und ökonomischen Prozesse und Entwicklungen ihnen zugrunde liegen, kann man in unterrichtlichen Zusammenhängen zu wichtigen Einsichten und komplexen Erkenntnissen über die jeweilige Gesellschaft gelangen (vgl. LOHFERT/SCHWECKENDIEK: 1989, Vorwort). Dies soll an einem Beispiel deutlich gemacht werden: Das landeskundliche Lehrbuch „Blick auf Deutschland“ (KIRCHMEYER: 1997, 139) zeigt unter der provozierenden Überschrift „Stirbt die Familie?" vier Bilder aus verschiedenen Werbeanzeigen, die ,jeweils eine (in den Augen von Werbefachleuten), typische’ Familie zeigen“. Auch wenn auf zwei der Bilder Familien mit nur einem Kind zu sehen sind, entsprechen die präsentierten glücklichen und intakten Familien sicherlich nicht überall und uneingeschränkt der deutschen Realität - auch nicht der der 1990er Jahre. Es sind also keine Bilder für einen Unterricht zum Thema "So verhält sich eine deutsche Familie“ oder „So sieht 
eine deutsche Familie aus“, sondern vielmehr zu der Frage nach Familienbildern in Deutschland, wie sie als ideale Wunschvorstellung existieren. Im landeskundlich orientierten Fremdsprachenunterricht kann der Frage nachgegangen werden, wie es zu diesen Idealbildern kommt, welche Entwicklungen diese Vorstellungen, die ja als gesellschaftliche Muster zu verstehen sind, hervorgebracht haben.

Nun ist es aber durchaus so, dass Werbung nicht nur die vorhandenen Idealbilder und Wunschvorstellungen aufgreift und für ihre Zwecke nutzt; sie trägt auch dazu bei, dass in den Köpfen vieler Menschen derartige Idealbilder existieren, welche der Realität nicht entsprechen. Denn durch Werbung werden kollektive Vorstellungen und Leitbilder geprägt. Wenn nun die oberflächlich dargestellte ,heile Welt' in Beziehung gesetzt wird mit dem jeweiligen historischen Kontext der Werbung und als Idealbild, als Wunschvorstellung entschlüsselt wird, dann erst konkretisiert sich der landeskundliche Wert von Werbung. Nur in diesem Sinne ist MACAIRE/HOSCH $(1996,178)$ zuzustimmen, wenn sie postulieren, dass Werbung ein auf die jeweilige Entstehungszeit bezogenes „aktuelles Bild von Kultur- und Gesellschaft eines Landes“ vermittle. Und nur so kann der „ungewöhnliche Blick auf gesellschaftliche Prozesse und in individuelle Lebensräume" entstehen, den OCHMANN (2001, 4) dem landeskundlichen Lernen im Spiegel von Werbung zuschreibt. Wenn dann als weiterer Schritt eine Kontrastierung der eigenen und fremden Welt mithilfe von Werbeanzeigen gelingt, wird auch das Potenzial von Werbung im Hinblick auf interkulturelles Lernen angesprochen. Dieses Potenzial wird beispielsweise auch in dem bereits erwähnten Lehrbuch „Blick auf Deutschland“ (KIRCHMEYER: 1997, 139) in Ansätzen genutzt, wenn zu den gezeigten Familienbildern gefragt wird: „Könnte man diese Fotos auch für Werbeanzeigen in Ihrem Land verwenden? [...] Wenn nicht: Wie sehen ,Werbefamilien' in Ihrem Land aus?" Die hier eingesetzte Methode des interkulturellen Vergleichs mit dem Ziel des interkulturellen Lernens lässt sich natürlich auch bei der Betrachtung und Analyse von historischen Werbeanzeigen, die entweder mit aktuellen Werbeanzeigen aus der Zielkultur bzw. mit eigenkulturellen Werbeanzeigen verglichen werden können, gewinnbringend nutzen. Denn Werbung ist ,ein Spiegel der Kultur, der sie entstammt und auf die sie zielt" (SCHRÖDER: 2001, 199), gleichzeitig aber auch ein Spiegel ihrer Zeit. Werbung ist, so NORD $(1996,61)$ „in ihrem jeweiligen spezifisch historischkulturellen Kontext eingebettet" und muss vor diesem spezifischen Hintergrund gesehen werden.

Das erhebliche Potenzial für interkulturelles Lernen, das der Werbung eigen ist, wird umso greifbarer, hält man sich vor Augen, dass Werbung weitgehend mit Stereotypen, die meist Bilder vom ,Eigenen' und häufig aber auch Bilder des ,Fremden' betreffen. Diese Bilder kritisch zu hinterfragen und zu entschlüsseln führt zu einer interkulturellen Perspektive, die weit über einen faktisch orientierten Landeskundeunterricht mit dem Ziel der Vermittlung von Realien hinausgeht. Denn ein interkulturell ausgerichteter Landeskundeunterricht mit Werbematerial arbeitet 
weniger mit Fakten und konkreten Realien, sondern vielmehr mit Repräsentationen von fremdkulturellen Bildern, Idealen, Werten und Normen, die in Beziehung gesetzt werden müssen, zu den entsprechenden eigenkulturellen Aspekten. Und natürlich geht es auch um die Emotionen, die durch die auf eine affektive Wirkung abzielende Werbung angesprochen und ausgelöst werden. In der Bewertung dieser Emotionen und ihrer Angemessenheit existieren erhebliche Unterschiede, die kulturell determiniert sind.

Für HENNECKE (1999, 2) steht fest: „Gerade Werbung ist heute ein wichtiges Instrument, um symbolische Bedeutungen, gesellschaftliche und kulturelle Werte, Normen und Leitbilder und damit Ideologie zu vermitteln." Wenn Hennecke das Wort „heute“ betont, dann spricht er sicherlich eine wichtige Entwicklungstendenz moderner Werbung an, die Anfang der 1990er Jahre einsetzt und die im Hinblick auf landeskundliches und interkulturelles Lernen mit Werbematerial äußerst bedeutsam erscheint. Waren in früheren Jahren die meisten Werbespots „eher platte, in schlichte Szenarien gepackte Produktanpreisungen und Kaufappelle", lässt sich „am Beginn der 1990er Jahre [...] in der Entwicklung des Werbespots jedoch eine Aufladung mit symbolischen und mythischen Subtexten und eine Verschiebung weg von ,sachlicher' und hin zu ,imaginativer Referenz" (KLOEPFER/LANDBECK: 1991, 96 ff.) feststellen. Mehr als in früheren Werbezeiten sind aktuelle Werbetexte ein Spiel mit kulturellen Symbolen, mit Elementen des kollektiv-kulturellen Gedächtnisses einer Gesellschaft, mit kollektiven Mythen, die neu oder umerzählt werden. Diese Veränderung lässt sich auch dadurch erklären, dass heutige Werbung, will sie ,funktionieren' und ihr Ziel erreichen, sich verstärkt als fiktionales Imaginationsangebot und als Vermittler von gesellschaftlichen Leitbildern und Lebensstilen präsentieren muss, während in älteren Werbetexten häufig das Informationsangebot über das Imaginationsangebot dominierte. Die Entschlüsselung dieses Imaginationsangebotes und der Vergleich mit eigenkulturellen Werbetexten kann interkulturelles Lernen auf eine interessante Weise in Gang setzen.

Eine weitere Entwicklung ist in diesem Zusammenhang von Bedeutung: Seit den 1990er Jahren bis zur Gegenwart ging der Trend in der Werbewirtschaft zu international ausgerichteten und standardisierten Werbekampagnen. Das heißt, dass man insbesondere aus Kostengründen das Ziel verfolgt, die Werbebotschaft eines international vermarkteten Produkts in verschiedenen Ländern beizubehalten, ohne das Thema oder die formale Umsetzung der Werbebotschaft zu verändern, so dass eine Werbekampagne weltweit eingesetzt werden kann (vgl. MONTES FERNANDEZ: 2003). Lediglich in sprachlicher Hinsicht wird der Werbetext dann in Form einer Übersetzung adaptiert, wenn nicht ohnehin Englisch benutzt wird. Diese Standardisierung wurde und wird in der Werbewirtschaft immer mehr kontrovers diskutiert, und die Vertreter der standardisierten Werbung nehmen kontinuierlich ab, nachdem man mehr und mehr erkannt hat, dass Konsummuster und Konsumverhalten soziokulturell gebunden sind und von Kultur zu Kultur, 
von Gesellschaft zu Gesellschaft stark voneinander abweichen. Da jedoch gerade diese Aspekte für die Rezeption und die Akzeptanz einer Werbekampagne verantwortlich seien, müssten standardisierte Werbemittel, die um alle mutmaßlich kulturspezifischen Elemente reduziert sind, ihre Wirkung verfehlen. Dagegen gehen Befürworter standardisierter Werbekampagnen „von einer Homogenisierung der Kulturen in industrialisierten Gesellschaften aus, in der die kulturellen Konvergenzen zur Überwindung von interkulturellen Unterschieden führen“ (MONTES FERNANDEZ: 2003). Lebensstile und Lebensbedingungen, aber auch Werte und Einstellungen seien in unserer globalisierten Realität dermaßen vereinheitlicht, dass eine kulturelle Differenzierung der Werbung nicht mehr nötig sei, zumal Werbung als bedeutender Bestandteil des globalen Netzwerks selbst zur Homogenisierung beitrage. MÜLLER $(1997,7)$ beschreibt die Argumente der Befürworter folgendermaßen: „Die Welt ist ein zusammenhängendes Netzwerk geworden, in dem Bedeutungen zwischen unterschiedlichen Kulturen ebenso ausgetauscht werden wie Güter und Menschen [...]. Es lassen sich ähnliche kulturelle Veränderungen, bspw. die Rolle der Frau in der Gesellschaft oder der Freizeitorientierung betreffend, feststellen [...]. Es wird die Ansicht vertreten, daß Werte und Einstellungen in industrialisierten Gesellschaften konvergieren [...]. Durch diese Entwicklung könnte eine erfolgreiche Standardisierung gewährleistet werden. Die Befürworter einer Standardisierung gehen davon aus, dass kulturelle Faktoren, die eine Standardisierung von Werbung beeinträchtigen könnten, obsolet geworden sind [...]. In einer globalen Kultur existiere eine interkulturell ähnliche Erfahrungswelt, auf die die Werbung Bezug nehmen kann. " MÜLLER diskutiert in seiner werbewirtschaftlichen Untersuchung ausführlich verschiedene Ansätze der Wirtschaftswissenschaften. Er kommt zu dem Schluss, dass sich trotz des intensiver werdenden Kulturaustauschs eine interkulturelle Homogenisierung, die eine interkulturell standardisierte Werbung ermöglichen würde, nicht abzeichne. Besonders der ikonische Kode der Bilder sei häufig nicht interkulturell übertragbar, nicht zuletzt wegen des Vergangenheitsbezugs aller Kulturen, der trotz allem Kulturaustausch bestehen bleibe. Die Fragestellung, ob eine fremdkulturelle Werbung in der eigenen Kultur seiner Funktion gerecht werden kann oder warum eventuell eine global ausgerichtete Werbekampagne in der Zielkultur oder im eigenen gesellschaftlichen Kontext nicht funktioniert, kann zu interessanten Einsichten führen.

Abschließend sei gesagt, dass die auch in einer globalisierten Welt vorhandene Kulturgebundenheit von Werbung sich meiner Ansicht nach äußerst gewinnbringend für Prozesse des interkulturellen Lernens ins Spiel bringen lässt, wenn es gelingt, eine Kontrastierung bzw. eine Verbindung von Eigenem und Fremdem herzustellen. Dabei ist auch hier wiederum ein Bogen zur Sprache der Werbung herstellbar. Darauf weist MONTES FERNANDEZ (2003) hin, wenn sie schreibt: „Die Sprache der Werbung reflektiert aufgrund ihrer spezifischen linguo-stilistischen 
Merkmale die interkulturellen Konventionen der verschiedenen Ausdrucksformen der Emotionalität und Suggestivität in den einzelnen Kulturen.“

\section{Literatur:}

HENNECKE (1999): Hennecke, Angelika. Im Osten nichts Neues? Eine pragmalinguistischsemiotische Analyse ausgewäblter Werbeanzeigen für Ostprodukte im Zeitraum 1993 bis 1998. Frankfurt: Lang, 1999.

HURRELMANN (2000): Hurrelmann, Bettina. Nie waren sie wertvoller als heute, in: Praxis Deutsch, 2000, 27, Nr. 163, 16-25.

KIRCHMEYER (1997): Kirchmeyer, Susanne. Blick auf Deutschland. Erlesene Landeskunde. Stuttgart u.a.: Ernst Klett, 1997.

KLOEPFER/LANDBECK (1991): Kloepfer, Rolf/Hanne Landbeck. Ästhetik der Werbung. Der Fernsehspot in Europa als Symptom neuer Macht. Frankfurt/M.: Fischer, 1991.

KOLLMANN (1998): Kollmann, Karl. Werbung - Überblick, Kritik und Fakten. Ein kleiner konsumwissenschaftlicher Rückblick, in: ide - Informationen zur Deutschdidaktik, 1998, 22, Nr. 3, 18-28.

LOHFERT/SCHWECKENDIEK (1989): Lohfert, Walter/Schweckendiek, Jürgen. Werbung und Anzeigen II. München: Goethe-Institut, 1989.

MACAIRE/HOSCH (1996): Macaire, Dominique/Hosch, Wolfram. Bilder in der Landeskunde. Berlin u.a.: Langenscheidt, 1996.

MAIWALD (2005): Maiwald, Klaus. Wahrnehmung - Sprache - Beobachtung. Eine Deutschdidaktik bilddominierter Medienangebote. München: kopaed, 2005.

MONTES FERNANDEZ (2003): Montes Fernandez, Antonia. Die interkulturelle Dimension von Werbeanzeigen - eine übersetzungsrelevante kontrastive Textanalyse, in: Translation Journal, 2003, 7, Nr. 2 [http://accurapid.com/journal/24werbung.htm, Stand: 1. 11. 2009].

MÜLLER (1997): Müller, Wendelin G. Interkulturelle Werbung. Heidelberg: Physica, 1997.

NORD (1996): Nord, Holger. Werbung als Spiegel der Gesellschaft, in: InfoDaF, 1996, 23, Nr. 1, 61-68.

OCHMANN (2001): Ochmann, Nana. Werbung in Deutschland. Eine Ausstellung des GoetheInstituts in Zusammenarbeit mit dem Deutschen Werbemuseum und dem stern. Materialien. München: Goethe-Institut, 2001.

RÖMER (1973): Römer, Ruth. Die Sprache der Anzeigenwerbung. Düsseldorf: Schwann, 3. Aufl., 1973.

SCHRÖDER (2001): Schröder, Hartmut. Urjala und Leppävirta: Fallstudien zum Finnlandbild in der deutschen Werbung, in: Hahn, Martin/Ylönen, Sabine (Hg.): Werbekommunikation im Wandel. Frankfurt/M. u.a.: Lang, 2001, 199-209. 